\title{
Guidance Laws with Finite Time Convergence
}

\author{
Di Zhou* and Sheng Sun‡ \\ Harbin Institute of Technology, 150001 Harbin, People's Republic of China \\ and \\ Kok Lay Teo \\ Curtin University of Technology, Perth 6102, Australia \\ DOI: $\underline{10.2514 / 1.42976}$
}

\begin{abstract}
Conventional guidance laws are designed based on Lyapunov theorems on asymptotic stability or exponential stability. They will guide the line-of-sight angular rate to converge to zero or its small neighborhood, however, only as time approaches infinity. In this paper, new guidance laws with finite convergent time are proposed. The guidance laws are obtained based on new sufficient conditions derived in this paper for the finite time convergence of the line-of-sight angular rate. It is proved that, with the guidance laws, the line-of-sight angular rate will converge to zero or a small neighborhood of zero before the final time of the guidance process. Furthermore, such guidance laws will ensure finite time convergence and finite time stability in both the planar and three-dimensional environments. Simulation results show that the guidance laws are highly effective.
\end{abstract}

\section{Nomenclature}

$a_{M r}, a_{M \theta}, \quad=$ missile acceleration along the line-of-sight axes

$a_{M \phi}$

$a_{r}, a_{\theta}, a_{\phi}=$ relative acceleration along line-of-sight axes

$a_{T r}, a_{T \theta}, \quad=$ target acceleration along line-of-sight axes

$a_{T \phi}$

$N$

$q$

$\dot{q}$

$\ddot{q}$

$r$

$\dot{r}$

$\ddot{r}$

$t$

$u$

$u_{r}$

$V_{M}$

$V_{T}$

$w$

$w_{r}$

$x_{M}, y_{M}, z_{M}$

$x_{T}, y_{T}, z_{T}$

$\theta$

$\phi$

$\varphi_{M}$

$\varphi_{T}$

$\psi_{M}$

$\psi_{T}$

$=$

= line-of-sight angle

$=$ derivative of $q$ with respect to time

$=$ second-order derivative of $q$ with respect to time

$=$ relative range

$=$ derivative of $r$ with respect to time

$=$ second-order derivative of $r$ with respect to time

$=$ time

$=$ missile acceleration normal to line of sight

$=$ missile acceleration along line of sight

$=$ missile velocity

$=$ target velocity

target acceleration normal to line of sight

target acceleration along line of sight

$=$ position coordinates of missile in inertial frame

position coordinates of target in inertial frame

azimuth

= elevation

= flight-path angle of missile

= flight-path angle of target

$=$ heading angle of missile

$=$ heading angle of target

\section{Introduction}

$\mathbf{P}$ ROPORTIONAL navigation (PN) and its variants have been widely used as homing guidance laws because they are highly efficient and easy for implementation [1-10]. The PN guidance law

Received 29 December 2008; revision received 27 June 2009; accepted for publication 19 July 2009. Copyright (C) 2009 by the American Institute of Aeronautics and Astronautics, Inc. All rights reserved. Copies of this paper may be made for personal or internal use, on condition that the copier pay the $\$ 10.00$ per-copy fee to the Copyright Clearance Center, Inc., 222 Rosewood Drive, Danvers, MA 01923; include the code and $\$ 10.00$ in correspondence with the CCC.

*Professor, Department of Control Science and Technology, Mailbox 327; zhoud@hit.edu.cn.

${ }^{\dagger}$ Ph.D. Student, Department of Control Science and Technology, Mailbox 327.

†Professor, Department of Mathematics and Statistics, Building 314. has the required accuracy to intercept a nonmaneuvering target or a weakly maneuvering target. Further, a missile under a PN guidance law has to have advantages in both maneuverability and agility. However, for the task of intercepting a target with maneuverability close to that of a missile, PN guidance laws are unable to achieve the required precision.

An effective approach to deal with maneuverable targets is to apply a robust guidance scheme. Many existing robust guidance laws, such as $H_{\infty}$ guidance law [11], $L_{2}$ gain guidance law [12], Lyapunov-based nonlinear guidance law [13], and first-order slidingmode guidance laws [14-17] are obtained based on Lyapunov theorems on asymptotic stability or exponential stability. The crucial technique to designing the $H_{\infty}$ guidance law [11] was to find the analytic solution of the associated Hamilton-Jacobi partial differential inequality of the missile guidance problem. Then, the system describing the missile guidance problem was said to have a $L_{2}$ gain less than a given level. Because the $L_{2}$ gain is an index defined in a time horizon from zero to infinity, the $H_{\infty}$ guidance law is not a guidance law with finite time convergence, although it exhibited strong robustness against disturbances from the target's maneuvers and variations in initial engagement conditions. The $L_{2}$ gain guidance law [12] was also designed to satisfy the $L_{2}$ gain and not a guidance law with finite convergent time. In the design of the Lyapunov-based nonlinear guidance law [13], a compact set, into which the state of guidance system converges, was obtained by solving a linear matrix inequalities characterization of the pole placement problem. Certainly, the convergence rate can be adjusted by the pole selection. However, theoretically speaking, for nonmaneuvering targets and targets having a constant acceleration, only asymptotic stability was obtained and demonstrated. Existing firstorder sliding-mode guidance laws [14-17] were all designed with Lyapunov theorems on asymptotic stability or exponential stability such that they had not been proved to guarantee a finite time convergence. In short, the theoretical results only indicated that the line-of-sight (LOS) angular rate under the aforementioned guidance laws will converge to zero or a small neighborhood of zero as time approaches infinity. These theoretical findings are inconsistent with practical observations. In many applications, the time of termination is really quite short. For example, in the space interception where a missile is intercepting a ballistic target, sometimes the time of terminal guidance is only several seconds such that the guidance law is required to ensure finite time convergence of the LOS angular rate.

In recent years, the finite time stability for feedback control systems (i.e., the states of the systems converge to their equilibrium point and then stay there) has become an active research area. Finite time control, which is related to finite time stability, was first proposed in [18] in 1986. It has since generated many research activities 
in the following two decades; see, for example, [19-31]. The fundamental tool for analyzing the finite time stability of control systems is the second method of Lyapunov. Many related results are reported in the literature. Examples include 1) finite time stability based on Lyapunov and converse Lyapunov results involving scalar differential inequalities [21], 2) finite time stability of a guidance system [22], 3) arbitrary-order sliding-mode controls with finite time convergence [23], and 4) guidance laws with finite time convergence based on second-order sliding-mode controls [28-31]. For guidance laws with finite time convergence based on second-order slidingmode controls, they are naturally obtained by applying second-order sliding-mode controls to guidance law design, but rather complex in structure. In this paper, a guidance scheme with finite time convergence based on Lyapunov scalar differential inequality is proposed. Its complexity is just comparable to that of a firstorder sliding-mode guidance law. Moreover, the proposed guidance scheme covers the first-order sliding-mode guidance law [16]; in other words, the first-order sliding-mode guidance law is also able to guide the LOS angular rate to converge to zero in a finite time. To ensure finite time convergence of the LOS angular rate, the proposed guidance scheme will involve a nonsmooth signum function. In practical applications, we usually use a saturation function in lieu of the signum function for the purpose of removing the chattering.

In practice, an actual target-to-missile motion occurs in a threedimensional environment. In a spherical coordinate, the motion is governed by three second-order nonlinear and coupling differential equations. When the LOS angles and the LOS angular rates are small, the three-dimensional missile-target relative motion can be simplified into two planar relative motions, in which the cross couplings between the elevation and the azimuth are ignored. With such simplifications, the design and analysis of guidance laws become much easier. However, such an approach is ad hoc in nature.

Although many three-dimensional PN guidance laws for control systems described by equations of three-dimensional relative motion have been proposed and analyzed (see, for example, [32-37]), some major deficiencies remain. For example, such three-dimensional PN guidance laws are ineffective to overcome unfavorable influences of target maneuvers. Consequently, many guidance laws, which are robust against target maneuvers, are studied for systems described by the equations of the planar relative motion (see, for example, [11$17,28-31])$. However, no results on the design of guidance laws with finite time convergence in a three-dimensional environment are available in the literature.

In this paper, we consider a system described by the equations of a planar relative motion. Design methods for guidance laws with finite time convergence, and ensuring finite time stability of the guidance process, will be obtained in Sec. II. Then, the planar guidance laws with finite time convergence are applied to a three-dimensional engagement model. The finite time convergence of the LOS angular rates, in both the elevation loop and the azimuth loop, is established. Finally, some simulations are carried out. The simulation results show the effectiveness of the finite time convergent guidance laws so obtained.

\section{Planar Finite Time Convergent Guidance Laws}

\section{A. Equations of Planar Missile-Target Engagement}

The geometry of planar interception is depicted in Fig. 1 . The corresponding equations of motion are given by

$$
\begin{gathered}
\dot{r}=V_{T} \cos \left(q-\varphi_{T}\right)-V_{M} \cos \left(q-\varphi_{M}\right) \\
r \dot{q}=-V_{T} \sin \left(q-\varphi_{T}\right)+V_{M} \sin \left(q-\varphi_{M}\right)
\end{gathered}
$$

Differentiating Eqs. (1) and (2) with respect to time yields

$$
\begin{gathered}
\ddot{r}=r \dot{q}^{2}+w_{r}-u_{r} \\
\ddot{q}=-\frac{2 \dot{r}}{r} \dot{q}+\frac{1}{r} w-\frac{1}{r} u
\end{gathered}
$$

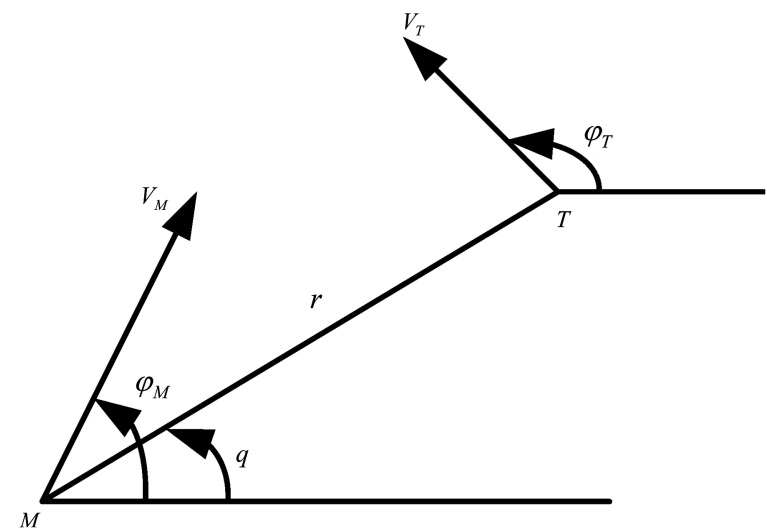

Fig. 1 Planar interception geometry.

By accepting the intuition that zeroing the LOS angular rate will lead to interception, a desired guidance law can be obtained as follows.

Achieving the convergence of $\dot{q}$ by adjusting $u$ and then defining $x=\dot{q}$, Eq. (4) can be written as

$$
\dot{x}(t)=-\frac{2 \dot{r}(t)}{r(t)} x(t)-\frac{1}{r(t)} u(t)+\frac{1}{r(t)} w(t)
$$

where the starting time of the guidance process is taken to be zero (i.e., $t=0$ ). The initial values of the state variables in the guidance system are denoted as $r(0), \dot{r}(0)$, and $x(0)$. At time $t$, they are denoted as $r(t), \dot{r}(t)$, and $x(t)$.

\section{B. Finite Time Stability of Nonlinear Systems}

In the design of finite time convergent guidance laws, we need some results on the finite time stability of nonlinear systems. The definition of finite time stability for a time-invariant nonlinear system given in [25] is extended to a time-varying nonlinear system as follows.

Definition: Consider a nonlinear system in the form of

$$
\dot{x}=f(x, t), \quad f(0, t)=0, \quad x \in R^{n}
$$

where $f: U_{0} \times R \rightarrow R^{n}$ is continuous on $U_{0} \times R$, and $U_{0}$ is an open neighborhood of the origin $x=0$. The state of the system is said to converge to its local equilibrium $x=0$ in finite time if, for any given initial time $t_{0}$ and initial state $x\left(t_{0}\right)=x_{0} \in U$, there exists a settling time $T \geq 0$, which is dependent on $x_{0}$, such that every solution of the system (6) $) x(t)=v\left(t ; t_{0}, x_{0}\right) \in U /\{0\}$, satisfies

$$
\begin{cases}\lim _{t \rightarrow T\left(x_{0}\right)} v\left(t ; t_{0}, x_{0}\right)=0, & t \in\left[t_{0}, T\left(x_{0}\right)\right) \\ v\left(t ; t_{0}, x_{0}\right)=0, & t \geq T\left(x_{0}\right)\end{cases}
$$

Moreover, if the system (local) equilibrium $x=0$ is Lyapunov stable with finite time convergence in a neighborhood of the origin $U \subset U_{0}$, then the system equilibrium is called finite time stable. If $U=R^{n}$, then the origin is a global finite time stable equilibrium.

The following lemma provides a useful result for the study of finite time convergent guidance laws.

Lemma 1: Consider the nonlinear system described by Eq. (6). Suppose that there is a $C^{1}$ (continuously differentiable) function $V(x, t)$ defined in a neighborhood $\widehat{U} \subset R^{n}$ of the origin, and that there are real numbers $\alpha>0$ and $0<\lambda<1$, such that $V(x, t)$ is positive definite on $\widehat{U}$ and that $\dot{V}(x, t)+\alpha V^{\lambda}(x, t) \leq 0$ on $\widehat{U}$. Then, the zero solution of system (6) is finite time stable.

Proof: By assumption, $\dot{V}(x, t)+\alpha V^{\lambda}(x, t) \leq 0$ on $\hat{U}$. Thus, it is clear that

$$
\dot{V}(x, t) \leq-\alpha V^{\lambda}(x, t), \quad \forall t \geq 0
$$


Since $V(x, t)>0$ on $\widehat{U}$, solving Eq. (8) yields

$$
V^{1-\lambda}(x, t) \leq V^{1-\lambda}\left(x_{0}, 0\right)-\alpha(1-\lambda) t, \quad 0 \leq t \leq t_{r}
$$

and

$$
V(x, t)=0, \quad \forall t \geq t_{r}
$$

The settling time, depending on initial state $x_{0}$, is given by

$$
t_{r} \leq \frac{V\left(x_{0}, 0\right)^{1-\lambda}}{\alpha(1-\lambda)}
$$

Remark 1: Note that if $\widehat{U}=R^{n}$ and $V(x, t)$ is radially unbounded, then the origin is globally finite time stable.

\section{Finite Time Convergent Guidance Laws}

Based on the preceding finite time stability theory, we can prove sufficient conditions for the finite time convergence of the guidance system (5).

Theorem 1: Consider the guidance system (5). If there exists a control $u$ such that the system state satisfies

$$
x\left[\dot{x}+\frac{\beta|x|^{\eta} \operatorname{sgn} x}{r(t)}\right] \leq 0, \quad \forall t \geq 0
$$

where $\beta=$ const. $>0$ and $-1<\eta=$ const. $<1$, then the LOS angular rate $x$ converges to zero in finite time. The convergence rate increases as the value of $\beta$ is increased. Furthermore, under the situation that $|x(0)|<1 \mathrm{rad} / \mathrm{s}$, the convergence rate also increases as the value of $\eta$ is decreased.

Proof: Choose a continuously differentiable positive-definite function in the neighborhood of origin $\widehat{U} \subset R^{n}$ as

$$
V_{1}=x^{2}
$$

The derivative of $V_{1}$ along the trajectories of Eq. (12) satisfies

$$
\dot{V}_{1} \leq-\frac{2 \beta}{r(t)} V_{1}^{\frac{1+\eta}{2}}, \quad \forall t>0
$$

During the time horizon of the guidance process, we have

$$
\dot{r}(t)<0, \quad 0<r(t)<r(0), \quad \forall t>0
$$

Combining Eqs. (14) and (15), we obtain

$$
\dot{V}_{1}<-\frac{2 \beta}{r(0)} V_{1}^{\frac{1+\eta}{2}}, \quad \forall t>0
$$

According to the condition $-1<\eta=$ const. $<1$, there exists $0<(1+\eta) / 2<1$. Now, by Lemma 1 , the LOS angular rate $x$ converges to zero in finite time, and the settling time is given by

$$
t_{r 1}<\frac{|x(0)|^{1-\eta} r(0)}{\beta(1-\eta)}
$$

It is revealed in Eq. (17) that the convergence rate increases as the value of $\beta$ is increased. Moreover, in practice, the absolute value of the initial LOS angular rate $|x(0)|$ must be much less than $1 \mathrm{rad} / \mathrm{s}$, and so the convergence rate increases as the value of $\eta$ is increased. $\square$

A proper choice of $\beta$ will depend on whether the target acceleration $w$ will have been compensated in the succeeding guidance law design. If so, $\beta$ can be chosen as a relatively small constant; otherwise, $\beta$ should be relatively large so as to deal with the target acceleration and to ensure the finite time convergence of the LOS angular rate. However, in practical applications, $\beta$ should not be too large to induce the chattering.

Substituting Eq. (5) into Eq. (12) gives

$$
x\left[-\frac{2 \dot{r}(t)}{r(t)} x-\frac{u}{r(t)}+\frac{w}{r(t)}+\frac{\beta|x|^{\eta} \operatorname{sgn} x}{r(t)}\right] \leq 0
$$

Thus, by choosing the guidance law as given by

$$
u=-N \dot{r}(t) x+w+\beta|x|^{\eta} \operatorname{sgn} x, \quad N=\text { const. }>2
$$

we obtain the results presented in the following theorem.

Theorem 2: Consider the guidance system (5). The guidance law (19) nullifies the LOS angular rate in infinite time. The convergence rate of the LOS angular rate increases as the value of $\beta$ is increased and, furthermore, it also increases as the value of $\eta$ is decreased when $|x(0)|<1 \mathrm{rad} / \mathrm{s}$. A reasonable range of $\eta$ is $0 \leq \eta<1$. If the target-to-missile relative velocity is approximately a constant, the LOS angular rate, under the guidance law (19), converges to zero before the final time of the time horizon of the guidance process.

Proof: Substituting Eq. (19) into Eq. (5) yields

$$
\dot{x}=\frac{(N-2) \dot{r}(t)}{r(t)} x-\frac{\beta|x|^{\eta} \operatorname{sgn} x}{r(t)}
$$

Then, by substituting Eq. (20) into Eq. (12), we obtain

$$
x\left[\dot{x}+\frac{\beta|x|^{\eta} \operatorname{sgn} x}{r(t)}\right]=-\frac{(N-2)|\dot{r}(t)|}{r(t)} x^{2} \leq 0
$$

By Theorem 1, the LOS angular rate converges to zero in finite time and the convergence rate increases as the value of $\beta$ is increased and, furthermore, when $|x(0)|<1 \mathrm{rad} / \mathrm{s}$, it also increased as the value of $\eta$ is decreased.

In view of Eq. (19), we see that, if $-1<\eta<0$, there exists a singularity at $x=0$. Hence, a reasonable range of $\eta$ is $0 \leq \eta<1$. As $\eta=0$, the guidance law (19) is just the first-order slidingmode guidance law [16] which contains a proportional navigation guidance (PNG) term, a compensation term for the target acceleration, and a variable structure term. This fact indicates that the firstorder sliding-mode guidance law is also a guidance law with finite time convergence. Moreover, in the guidance law (19), the target acceleration $w$ has been compensated, so that even if $\beta$ was chosen as a relatively small constant, the guidance law would ensure the finite time convergence of the LOS angular rate.

Suppose that the target-to-missile relative velocity is approximately a constant, say,

$$
\dot{r}(t)=-c, \quad c=\text { const. }>0
$$

Then, at time $t$, the relative range can be calculated as

$$
r(t)=r(0)-c t
$$

Combining Eqs. (21) and (23) produces

$$
x\left[\dot{x}+\frac{\beta|x|^{\eta} \operatorname{sgn} x}{r(0)-c t}\right] \leq 0
$$

Solving Eq. (24), we obtain the settling time of $x$ when it converges to zero as follows:

$$
t_{r 2} \leq \frac{r(0)}{c}\left[1-e^{-\frac{c}{\beta(1-\eta)}|x(0)|^{1-\eta}}\right]
$$

Define the final time of the time horizon of the guidance process as $t_{f}$. Then, $r\left(t_{f}\right) \ll r(0)$ and the following equation holds:

$$
t_{f}=\frac{r(0)-r\left(t_{f}\right)}{c} \approx \frac{r(0)}{c}
$$

Comparing Eq. (25) with Eq. (26), we obtain

$$
t_{r 2}<t_{f}
$$

Equation (25) shows that the convergence rate of the LOS angular rate increases as the value of $\beta$ is increased and, furthermore, it also increases as the value of $\eta$ is decreased when $|x(0)|<1 \mathrm{rad} / \mathrm{s}$. 
In practical applications, the target acceleration $w$ is unknown and is usually difficult to estimate, but its upper bound can be estimated a priori. Suppose the target acceleration satisfies

$$
\|w\|_{\infty} \leq f, \quad f=\text { const. }>0
$$

where $\|w\|_{\infty}$ is the maximum value of $|w|$. Then, by appropriate modification of the guidance law (19), we obtain the results presented in following theorem.

Theorem 3: Consider the guidance system (5). The following guidance law

$$
\begin{gathered}
u=-N \dot{r}(t) x+f \operatorname{sgn} x+\beta|x|^{\eta} \operatorname{sgn} x \\
N=\text { const. }>2, \quad 0 \leq \eta<1
\end{gathered}
$$

nullifies the LOS angular rate in finite time. The convergence rate of the LOS angular rate increases as the value of $\beta$ is increased and the value of $\eta$ (when $|x(0)|<1 \mathrm{rad} / \mathrm{s}$ ) is decreased. If the target-tomissile relative velocity is approximately a constant, then the LOS angular rate, under the guidance law (29), converges to zero before the final time of the time horizon of the guidance process.

Proof: Substituting Eq. (29) into Eq. () gives

$$
\dot{x}=\frac{(N-2) \dot{r}(t)}{r(t)} x-\frac{\left(f+\beta|x|^{\eta}\right) \operatorname{sgn} x-w}{r(t)}
$$

Then, by substituting Eq. (으) into Eq. (느), it follows from Eqs. (15) and $(\underline{28)}$ that

$$
x\left[\dot{x}+\frac{\beta|x|^{\eta} \operatorname{sgn} x}{r(t)}\right]=\frac{(N-2) \dot{r}(t)}{r(t)} x^{2}-\frac{\varepsilon \operatorname{sgn} x-w}{r(t)} x \leq 0
$$

The remaining proof is similar to that given in the proof of Theorem 2, and hence is omitted here.

Now, we have proposed a planar finite time convergent guidance scheme. However, because most practical guidance processes are applied in a three-dimensional environment, we will study whether the planar guidance scheme will ensure the finite time convergence of the LOS angular rates when it is applied to the equations of threedimensional relative motion.

\section{Three-Dimensional Finite Time Convergent Guidance Laws}

\section{A. Formulation of a Three-Dimensional Missile-Target Engagement}

Consider the spherical LOS coordinates $(r, \theta, \phi)$ with origin fixed at the missile's gravity center. Let $\left(e_{r}, e_{\theta}, e_{\phi}\right)$ be the unit vectors along the coordinate axes (see Fig. 2). By virtue of the principles of kinematics, the three relative acceleration components $\left(a_{r}, a_{\theta}, a_{\phi}\right)$ can be expressed by the following set of second-order nonlinear differential equations [프-35]:

$$
\begin{gathered}
\ddot{r}-r \dot{\phi}^{2}-r \dot{\theta}^{2} \cos ^{2} \phi=a_{T r}-a_{M r} \equiv a_{r} \\
r \ddot{\theta} \cos \phi+2 \dot{r} \dot{\theta} \cos \phi-2 r \dot{\phi} \dot{\theta} \sin \phi=a_{T \theta}-a_{M \theta} \equiv a_{\theta} \\
r \ddot{\phi}+2 \dot{r} \dot{\phi}+r \dot{\theta}^{2} \sin \phi \cos \phi=a_{T \phi}-a_{M \phi} \equiv a_{\phi}
\end{gathered}
$$

The purpose of designing a guidance law is to nullify the LOS angular rates $\dot{\theta}$ and $\dot{\phi}$. To design such a guidance law, we consider Eqs. ( $\underline{32 \mathrm{~b}})$ and ( $\underline{32 \mathrm{c})}$. Obviously, there exist cross couplings between them. Define state variables as $x_{1}=\dot{\theta}, x_{2}=\dot{\phi}$ and control variables as $u_{1}=a_{M \theta}, u_{2}=a_{M \phi}$. Then, the coupling equations of the LOS motion can be rewritten as

$$
\begin{gathered}
\dot{x}_{1}=-\frac{2 \dot{r}}{r} x_{1}+2 x_{1} x_{2} \tan \phi-\frac{u_{1}}{r \cos \phi}+\frac{a_{T \theta}}{r \cos \phi} \\
\dot{x}_{2}=-\frac{2 \dot{r}}{r} x_{2}-x_{1}^{2} \sin \phi \cos \phi-\frac{u_{2}}{r}+\frac{a_{T \phi}}{r}
\end{gathered}
$$

During the time horizon of the guidance process, $x_{1}$ and $x_{2}$ are small variables. When $\phi$ is also a small variable, it gives $\phi \approx 1$. By omitting the third-order small amounts in Eqs. (33a) and (33b), they decoupled into

$$
\dot{x}_{1}=-\frac{2 \dot{r}}{r} x_{1}-\frac{u_{1}}{r}+\frac{a_{T \theta}}{r}
$$

and

$$
\dot{x}_{2}=-\frac{2 \dot{r}}{r} x_{2}-\frac{u_{2}}{r}+\frac{a_{T \phi}}{r}
$$

Comparing Eq. (34) with Eq. (5), we find that the decoupled threedimensional LOS angular motion is equivalent to two planar LOS angular motions.

By virtue of the results obtained in Sec. II, we note that, if the target acceleration can be estimated, then the two planar finite time convergent guidance laws can be designed as

$$
\begin{aligned}
& u_{1}=-N \dot{r} x_{1}+a_{T \theta}+\beta\left|x_{1}\right|^{\eta} \operatorname{sgn} x_{1} \\
& u_{2}=-N \dot{r} x_{2}+a_{T \phi}+\beta\left|x_{2}\right|^{\eta} \operatorname{sgn} x_{2}
\end{aligned}
$$

where $N=$ const. $>2, \beta=$ const. $>0$, and $0 \leq \eta=$ const. $<1$.

Suppose that only the upper bound of the target acceleration is available. Then, the two planar finite time convergent guidance laws can be designed as

$$
\begin{aligned}
& u_{1}=-N \dot{r} x_{1}+f_{1} \operatorname{sgn} x_{1}+\beta\left|x_{1}\right|^{\eta} \operatorname{sgn} x_{1} \\
& u_{2}=-N \dot{r} x_{2}+f_{2} \operatorname{sgn} x_{2}+\beta\left|x_{2}\right|^{\eta} \operatorname{sgn} x_{2}
\end{aligned}
$$

where $N=$ const. $>2, \beta=$ const. $>0$, and $0 \leq \eta=$ const. $<1$.

\section{B. Finite Time Convergence in Three-Dimensional Model}

For the guidance law (35), we have the following theorem.

Theorem 4: Consider the nonlinear system (33). The equations of three-dimensional relative motion, under the guidance law (35), nullify the LOS angular rates in finite time. The convergence rate of the LOS angular rate increases as the value of $\beta$ is increased and, furthermore, it also increases as the value of $\eta$ is decreased when $x_{1}^{2}(0) \cos ^{2} \phi(0)+x_{2}^{2}(0)<1 \mathrm{rad} / \mathrm{s}$.

Proof: By choosing an appropriate inertial reference coordinate system, we can ensure that $-0.5 \pi<\phi<0.5 \pi$. Thus, there exists a $\phi$ such that $\cos \phi>0$. Substituting Eq. (35) into Eq. (33) gives 


$$
\begin{gathered}
\dot{x}_{1}=\frac{(N / \cos \phi-2) \dot{r}}{r} x_{1}+2 x_{1} x_{2} \tan \phi-\frac{\beta\left|x_{1}\right|^{\eta} \operatorname{sgn} x_{1}}{r \cos \phi} \\
\dot{x}_{2}=\frac{(N-2) \dot{r}}{r} x_{2}-x_{1}^{2} \sin \phi \cos \phi-\frac{\beta\left|x_{2}\right|^{\eta} \operatorname{sgn} x_{2}}{r}
\end{gathered}
$$

Construct a continuously differentiable positive-definite function as

$$
V_{2}=x_{1}^{2} \cos ^{2} \phi+x_{2}^{2}
$$

Differentiating $V_{2}$ with respect to time along the trajectories of Eq. (37) gives

$$
\begin{aligned}
\frac{1}{2} \dot{V}_{2} & =\frac{(N / \cos \phi-2) \dot{r}}{r} x_{1}^{2} \cos ^{2} \phi-\frac{\beta\left|x_{1}\right|^{\eta+1}}{r} \cos \phi \\
& +\frac{(N-2) \dot{r}}{r} x_{2}^{2}-\frac{\beta\left|x_{2}\right|^{\eta+1}}{r} \leq-\frac{\beta}{r}\left(\left|x_{1}\right|^{\eta+1} \cos \phi+\left|x_{2}\right|^{\eta+1}\right) \\
& \leq-\frac{\beta}{r}\left(\left|x_{1} \cos \phi\right|^{\eta+1}+\left|x_{2}\right|^{\eta+1}\right) \leq-\frac{\beta}{r}\left(x_{1}^{2} \cos ^{2} \phi+x_{2}^{2}\right)^{\frac{\eta+1}{2}} \\
& =-\frac{\beta}{r} V_{2}^{\frac{\eta+1}{2}}
\end{aligned}
$$

Taking into account Eq. (15), we obtain

$$
\dot{V}_{2} \leq-\frac{2 \beta}{r} V_{2}^{\frac{\eta+1}{2}} \leq-\frac{2 \beta}{r(0)} V_{2}^{\frac{\eta+1}{2}}
$$

By the application of Lemma 1, we conclude that the guidance system is finite time convergent and the settling time satisfies the inequality

$$
t_{r 3} \leq \frac{r(0) V_{2}(0)^{\frac{1-\eta}{2}}}{\beta(1-\eta)}, \quad V_{2}(0)=x_{1}^{2}(0) \cos ^{2} \phi(0)+x_{2}^{2}(0)
$$

where $x_{1}(0), x_{2}(0)$, and $\phi(0)$ are the system's initial states.

It is revealed in Eq. (41) that the convergence rate of the LOS angular rate increases as the value of $\beta$ is increased. In practice, the initial LOS angular rates of a terminal guidance process are small. In fact, $x_{1}(0)$ and $x_{2}(0)$ are much less than $1 \mathrm{rad} / \mathrm{s}$. Thus, $V_{2}(0)<1$ holds and the convergence rate increases as the value of $\eta$ is decreased.

For the interception of nonmaneuvering targets, the finite time convergent guidance law (35) can be rewritten as

$$
u_{1}=-N \dot{r} x_{1}+\beta\left|x_{1}\right|^{\eta} \operatorname{sgn} x_{1} \quad u_{2}=-N \dot{r} x_{2}+\beta\left|x_{2}\right|^{\eta} \operatorname{sgn} x_{2}
$$

where $N=$ const. $>2, \beta=$ const. $>0$, and $0 \leq \eta=$ const. $<1$.

For intercepting maneuvering targets with acceleration being difficult to estimate in real time, the guidance law (36) is a finite time convergent guidance scheme in a three-dimensional interception.

Theorem 5: Consider the nonlinear system (33). If only the upper bounds of the target accelerations are available, that is, $f_{1} \geq\left\|a_{T \theta}\right\|_{\infty}$ and $f_{2} \geq\left\|a_{T \phi}\right\|_{\infty}$, where $f_{1}$ and $f_{2}$ are known constants, then the guidance law (36) guarantees the finite time convergence of the LOS angular rates. The convergence rate increases as the value of $\beta$ is increased and, furthermore, it increases as the value of $\eta$ is decreased when $x_{1}^{2}(0) \cos ^{2} \phi(0)+x_{2}^{2}(0)<1 \mathrm{rad} / \mathrm{s}$.

Proof: The proof is similar to that given for Theorem 4 , and hence is omitted.

In view of Theorems 4 and 5, we see that the planar finite time convergent guidance laws (35) and (36) ensure the finite time convergence of the LOS angular rates in the three-dimensional model.

In the guidance law $(36),-N \dot{r} x_{1}$ and $-N \dot{r} x_{2}$ are proportional navigation terms, whereas $f_{1} \operatorname{sgn} x_{1}$ and $f_{2} \operatorname{sgn} x_{2}$ are variable structure terms for dealing with the target accelerations. Moreover, the guidance law involves finite time convergent terms $\beta\left|x_{1}\right|{ }^{\eta} \operatorname{sgn} x_{1}$ and $\beta\left|x_{2}\right|^{\eta} \operatorname{sgn} x_{2}$. When $\eta=1$, the finite time convergent terms are transformed into proportional navigation terms. On the other hand, when $\eta=0$, they are transformed into variable structure terms, and Eq. (36) can then be rewritten as

$$
u_{1}=-N \dot{r} x_{1}+\varepsilon_{1} \operatorname{sgn} x_{1} \quad u_{2}=-N \dot{r} x_{2}+\varepsilon_{2} \operatorname{sgn} x_{2}
$$

where $\varepsilon_{1}=f_{1}+\beta>\left\|a_{T \theta}\right\|_{\infty}, \varepsilon_{2}=f_{2}+\beta>\left\|a_{T \phi}\right\|_{\infty}$.

For the case in which the finite time convergent guidance law (43) is equivalent to the first-order sliding-mode guidance law [16], if the gain of its variable structure term is larger than the maximum value of the target acceleration, the first-order sliding-mode guidance law steers the LOS angular rate to converge to zero in finite time, either in planar guidance or in three-dimensional guidance. The convergence rate increases as the gain of the variable structure term is increased.

The finite time convergent guidance laws, which are nonsmooth controllers, guarantee fast convergence and robustness of the guidance system. They involve a signum function, which indicates that the control variable will switch. In a practical system, the occurrence of a switching cannot be completely instantaneous. The delay of the occurrence of the switching induces the chattering effect. To remove the chattering, we may smoothen the signum function, usually replacing $\operatorname{sgn} x$ with a saturation function $\operatorname{sat}_{\delta}(x)$ expressed as

$$
\operatorname{sat}_{\delta}(x)= \begin{cases}1, & x>\delta \\ x / \delta, & |x| \leq \delta \\ -1, & x<-\delta\end{cases}
$$

where $\delta$ is small positive constant.

Remark 2: For Eq. (44), when $|x|>\delta$, $\operatorname{sat}_{\delta}(x)$ is equivalent to $\operatorname{sgn} x$. Thus, the guidance laws with finite time convergence guide the LOS angular rate to converge into a boundary layer $|x| \leq \delta$ in finite time.

\section{Simulation Results}

We investigate a space interception problem. Define an inertial reference coordinate system which is parallel to the coordinate system $M X Y Z$, as shown in Fig. 2. This system is inertially fixed and is centered at the launch site at the instant of the launch. In this system, the $X$ axis is taken to be in the horizontal plane and in the direction of the launch, the positive $Z$ axis is in the vertical plane, and the $Y$ axis is chosen in such a way that the coordinate system forms a right-handed coordinate system. The interceptor's initial position coordinates are $x_{M 0}=0 \mathrm{~m}, y_{M 0}=0 \mathrm{~m}$, and $z_{M 0}=0 \mathrm{~m}$. Its initial velocity is $V_{M 0}=3000 \mathrm{~m} / \mathrm{s}$ and its initial flight-path and heading angles are $\varphi_{M 0}=19 \mathrm{deg}$ and $\psi_{M 0}=56 \mathrm{deg}$, respectively. The target's initial position coordinates are $x_{T 0}=80 \mathrm{~km}, y_{T 0}=24 \mathrm{~km}$, and $z_{T 0}=40 \mathrm{~km}$. Its initial velocity is $V_{T 0}=7000 \mathrm{~m} / \mathrm{s}$ and its initial flight-path and heading angles are $\varphi_{T 0}=-25 \mathrm{deg}$ and $\psi_{T 0}=180 \mathrm{deg}$, respectively. It is easy to calculate the initial elevation and azimuth, $\theta_{0}=16.7 \mathrm{deg}$ and $\phi_{0}=25.6 \mathrm{deg}$, and their initial angular rates, $\dot{\theta}_{0}=0.0172 \mathrm{deg} / \mathrm{s}$ and $\dot{\phi}_{0}=0.015 \mathrm{deg} / \mathrm{s}$. Because these initial values are not small, there exist cross coupling effects between the elevation loop and the azimuth loop. The interceptor's maximum translation acceleration is $30 \mathrm{~m} / \mathrm{s}^{2}$, and the sampling period of its target seeker is $15 \mathrm{~ms}$. In both the elevation loop and the azimuth loop, we apply the finite time convergent guidance scheme. In the guidance laws with finite time convergence, it is required that $N>2$. Because $N$ is a parameter of the PNG component and we are quite familiar with it (usually $3 \sim 5$ ), we just set it to be three and investigate the other parameters $\beta$ and $\eta$. We simulate the following cases.

Case 1: Suppose that the target does not maneuver. We apply the guidance law (42) with $\beta=1$ and $\eta=0,0.05,0.2$, and 0.8 . The LOS angular rate and the missile acceleration in the elevation loop are plotted in Figs. $3 \mathrm{a}$ and $3 \mathrm{~b}$, respectively. The LOS angular rate and the missile acceleration in the azimuth loop are plotted in Figs. $4 \mathrm{a}$ and $4 \mathrm{~b}$, respectively. For all these various values of $\eta$, the miss distances $\overline{\text { are }}$ less than $0.01 \mathrm{~m}$.

It is clear from Figs. 3 and 4 that the LOS angular rates in both the elevation loop and the azimuth loop converge to zero in finite time, and the convergence rate increases as the value of $\eta$ is decreased. However, the chattering of the missile acceleration also increases as the value of $\eta$ is decreased. In particular, when $\eta=0$, the finite time convergence term converts to a variable structure term, causing the 


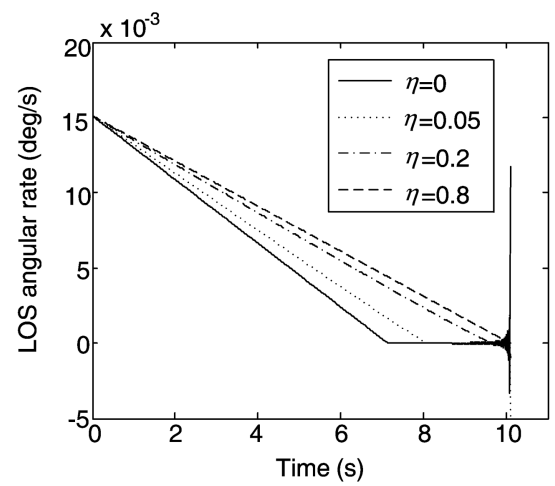

a)

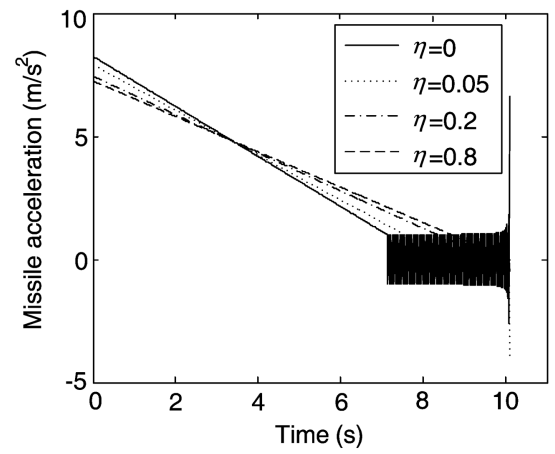

b)

Fig. 3 LOS angular rate and missile acceleration in elevation loop in case 1.

maximum chattering, as depicted in Figs. $3 \mathrm{~b}$ and $4 \mathrm{~b}$. The preceding results demonstrate that both the two planar finite time convergent guidance laws guarantee the finite time convergence of the LOS angular rates in the three-dimensional guidance process. Because the simulation results in the elevation loop and those in the azimuth loop

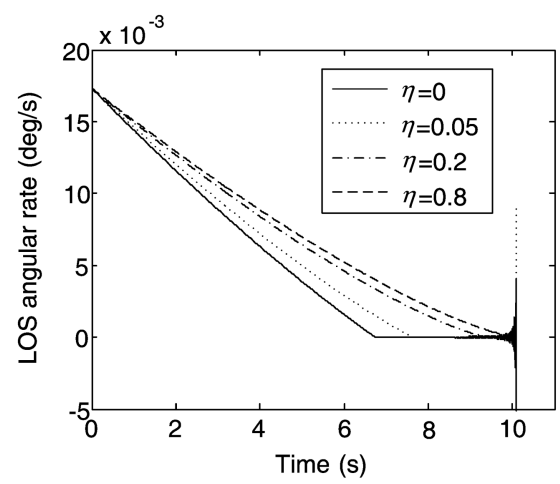

a)

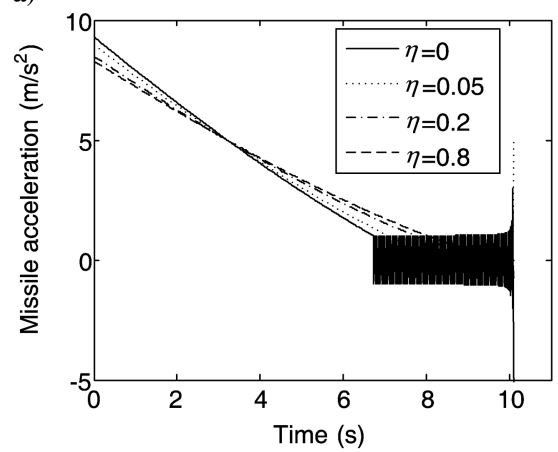

b)

Fig. 4 LOS angular rate and missile acceleration in azimuth loop in case 1 .
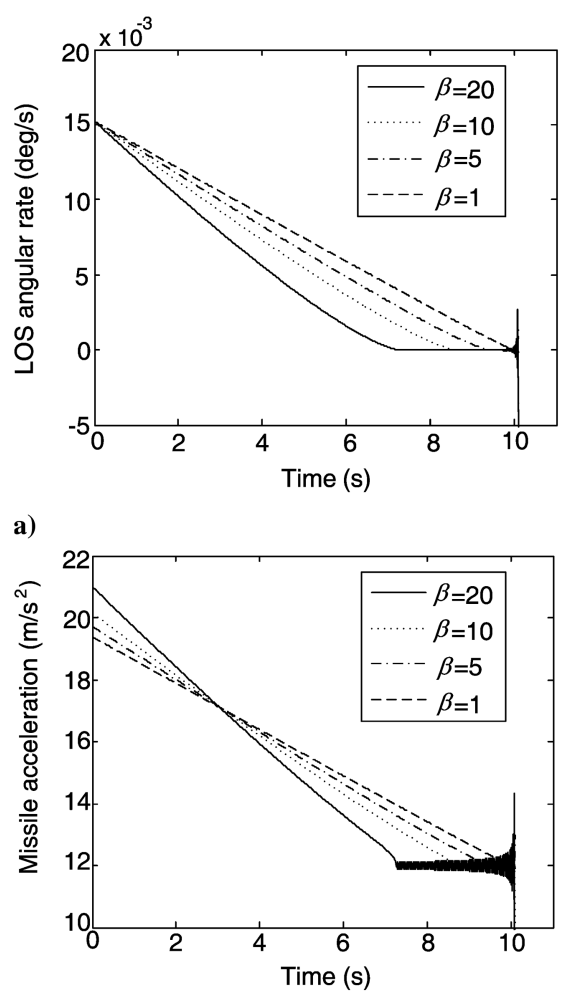

b)

Fig. 5 LOS angular rate and missile acceleration in elevation loop in case $2 \mathbf{a}$.

are similar, in the following cases, we only depict the results in the elevation loop.

Case 2a: Assume that the target escapes with constant acceleration components $a_{T \theta}=12 \mathrm{~m} / \mathrm{s}^{2}$ and $a_{T \phi}=12 \mathrm{~m} / \mathrm{s}^{2}$, and that these components can be estimated. Apply the finite time convergent

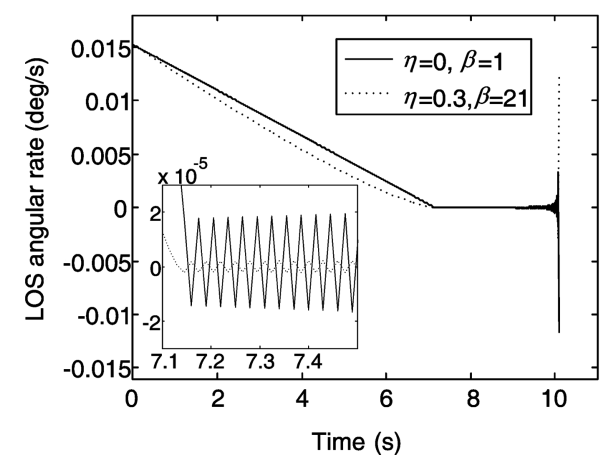

a)

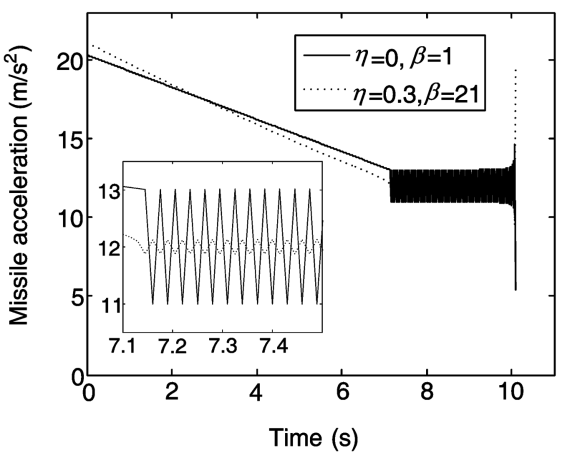

b)

Fig. 6 LOS angular rate and missile acceleration in elevation loop in case $2 b$. 


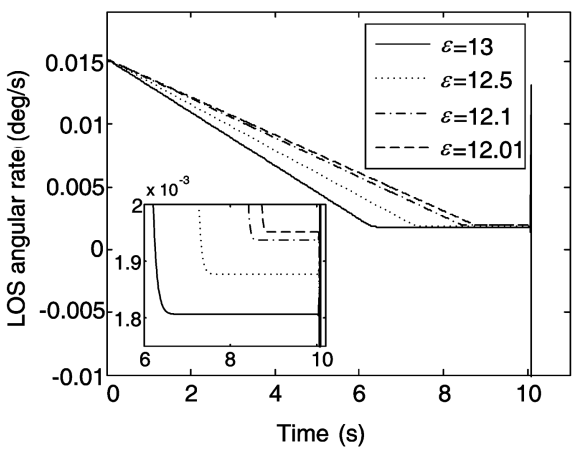

a)

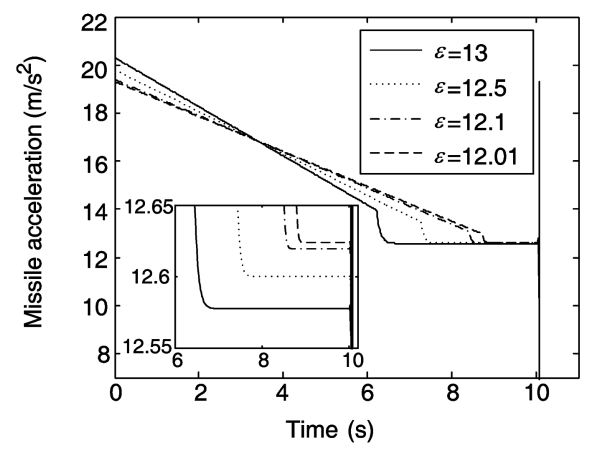

b)

Fig. 7 LOS angular rate and missile acceleration in elevation loop in case 3.

guidance law (35) with $\eta=0.3$ and $\beta=1,5,10$, and 20, respectively. The miss distances for all these different values of $\beta$ are less than $0.01 \mathrm{~m}$. The LOS angular rate and the missile acceleration in the elevation loop are plotted in Fig. 5. Figure 5a shows that the LOS angular rate converges to zero in finite time, which is before the final time, and the convergence rate increases as the value of $\beta$ is increased. However, it is apparent from Fig. $5 \mathrm{~b}$ that a large value of $\beta$ induces the chattering effect in the missile acceleration.

Case $2 \mathrm{~b}$ : The target maneuver is the same as that in case $2 \mathrm{a}$ and can be estimated. In the guidance law (35), we choose two groups of guidance parameters: for the first group, $\eta=0.3$ and $\beta=21$, and for the second group, $\eta=0$ and $\beta=1$. The miss distances for both these two groups of guidance parameters are less than $0.01 \mathrm{~m}$.

Figure 6a shows that the LOS angular rates, under the guidance law (35) with these two groups of parameters, converge with almost the same speed. However, Fig. $6 \mathrm{~b}$ shows that the chattering effect of the missile acceleration with $\bar{\eta}=0.3$ and $\beta=21$ is much less than that with $\eta=0$ and $\beta=1$. Hence, in a finite time convergent guidance process, we can ensure the required convergence rate while removing the chattering effect through a proper selection of the parameters $\eta$ and $\beta$.

Case 3: The target maneuver is the same as that in case 2 but cannot be estimated. Assume that the upper bound of the target acceleration can be found a priori. Then, we employ the finite time convergent guidance law (43). To reduce chattering, we replace the signum function $\operatorname{sgn} x$ with the saturation function $\operatorname{sat}_{\delta}(x)$ given by Eq. (44). Let $\delta=0.002 \mathrm{deg} / \mathrm{s}$ and $\varepsilon_{1}=\varepsilon_{2}=\varepsilon=12.01,12.1,12.5$, and $\overline{13}$, respectively. For these various values of $\varepsilon$, the miss distances are all

Table 1 Summary of miss distances in case $4 a$

\begin{tabular}{lcc}
\hline \hline Guidance law & & Miss distance, $\mathrm{m}$ \\
\hline & $\beta=13, \eta=0$ & 0.008 \\
Finite time & $\beta=13, \eta=0.02$ & 0.004 \\
Convergent & $\beta=13, \eta=0.1$ & 0.002 \\
Guidance & $\beta=13, \eta=0.4$ & 2.170 \\
& PN guidance & 4.476 \\
\hline \hline
\end{tabular}

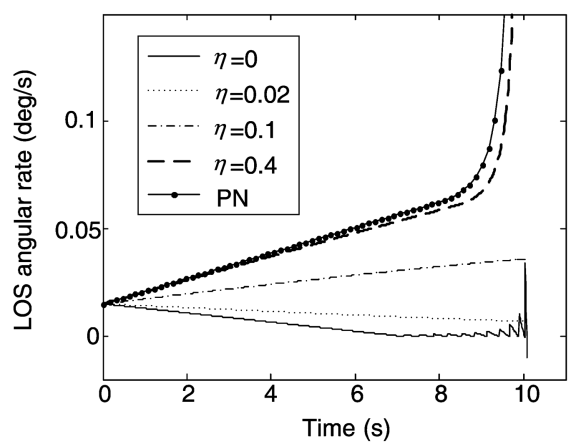

a)

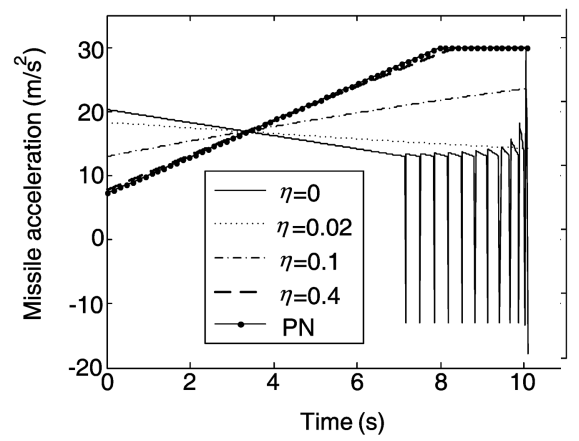

b)

Fig. 8 LOS angular rate and missile acceleration in elevation loop in case $4 a$.

less than $0.01 \mathrm{~m}$. The variations of the LOS angular rate and the missile acceleration in the elevation loop are plotted in Fig. 7.

In this case, the finite time convergent guidance law is just the firstorder sliding-mode guidance law. Because the gain of the variable structure term is larger than the maximum of the target acceleration, the LOS angular rate, as shown in Fig. 7a, converges into a prescribed boundary layer $|x| \leq \delta=0.002 \mathrm{deg} / \mathrm{s}$ (see subplots in Fig. 7a) before the final time of the guidance process and, furthermore, the convergence rate increases as the value of $\varepsilon$ is increased. From Fig. $\underline{7}$, we observe that the chattering effect is removed when the signum function is replaced by the saturation function.

Case 4a: Assume that the upper bound of the target acceleration is not known and that we cannot use a variable structure term to deal with the target maneuver. Even for such a case, the finite time convergent guidance scheme will still be robust to intercept the maneuvering target. This is because of the signum function in the guidance scheme, which not only ensures finite time convergence but is also effective on overcoming the bad influence of target acceleration. However, in this case, only when the parameter $\eta$ is small enough or the parameter is $\beta$ large enough, the guidance law will be robust enough to target accelerations and send the LOS angular rate to zero or a small boundary layer of zero. In fact, as $\eta$ approaches zero, the guidance law (42) tends to the first-order sliding-mode guidance law which is robust against target maneuvers and ensures the finite time convergence of LOS rate if the gain of its signum function is larger than the target acceleration.

Let the target acceleration be the same as that in case 2 . We use the finite time convergent guidance law (42), where there exists no compensation for target acceleration, and compare it with the PN guidance law $a_{M}=-N \dot{r} \dot{\phi}$ where $N=3$. In the guidance law (42),
Table 2 Summary of miss distances in case $4 \mathrm{~b}$

\begin{tabular}{lcc}
\hline \hline Guidance law & & Miss distance, $\mathrm{m}$ \\
\hline & $\beta=50, \eta=0.1$ & 0.013 \\
Finite time & $\beta=30, \eta=0.1$ & 0.002 \\
Convergent & $\beta=10, \eta=0.1$ & 0.003 \\
Guidance & $\beta=1, \eta=0.1$ & 2.908 \\
\hline \hline
\end{tabular}




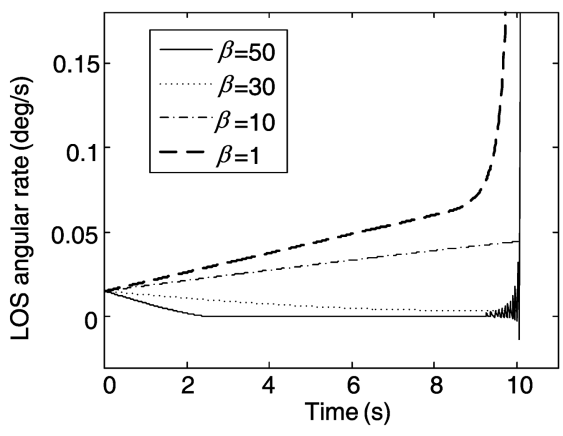

a)

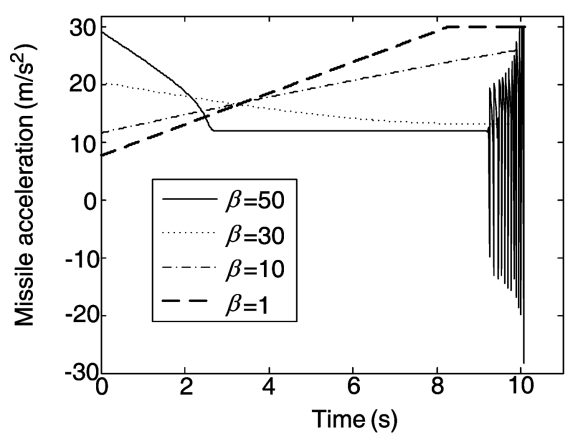

b)

Fig. 9 LOS angular rate and missile acceleration in elevation loop in case $4 b$.

$\beta=13$ and $\eta=0,0.02,0.1,0.4$, respectively. For $\beta=13$ and $\eta=0.1$, the miss distance is only $0.002 \mathrm{~m}$. On the other hand, the PN guidance law gives rise to a large miss distance of $4.476 \mathrm{~m}$. Table 1 summarizes the miss distances resulting from the finite time guidance scheme for different values of $\eta$ and that from the PN guidance law. Figure 8 illustrates the variations of the LOS angular rate and the missile acceleration in the elevation loop in case 4 a.

Case 4b: The target acceleration is the same as that in case $4 \mathrm{a}$. However, in the guidance law (42), we fix $\eta=0.1$, while letting $\beta=50,30,10$, and 1 , respectively. Table 2 summarizes the miss distances resulting from such a guidance law with these different values of $\beta$. Figure 9 illustrates the variations of the LOS angular rate and the missile acceleration in the elevation loop in case $4 \mathrm{~b}$.

The variations of the LOS angular rate and the missile acceleration under the PN guidance law can be seen from Fig. 8. At the beginning, the missile acceleration is small (see Fig. 8b), but due to the influence of the target maneuver, the LOS angular rate increases gradually (see Fig. 8a), moving to the eventual phase at which the missile acceleration is saturated (see Fig. 8b). On the other hand, the LOS angular rate diverges at an early time (see Fig. 8a). Therefore, the final miss distance under the PN guidance law is large. When $\beta$ is fixed, the variations of the LOS angular rate and the missile acceleration under the guidance law (42) can also be seen from Fig. $\underline{8}$. When the value of $\eta$ is large, the variations of the missile acceleration and the LOS angular rate are close to those under the PN guidance law. For example, when $\eta=0.4$, the LOS angular rate diverges early, resulting in a large miss distance. When the value of $\eta$ is small, the missile acceleration at the beginning is relatively large, ensuring the convergence of the LOS angular rate. The variations of the missile acceleration and the LOS angular rate are close to those under the first-order sliding-mode guidance law. However, if the value of $\eta$ is too small, because we have not used a saturation function in lieu of the signum function, the guidance law tends to induce the chattering effect, causing not only some increase in the miss distance but also unfavorable effects on the instruments onboard the missile.

When $\eta$ is fixed, the variations of the LOS angular rate and the missile acceleration under the guidance law (42) can be seen from Fig. 9. When the value of $\beta$ is small, the finite time convergent guidance law is close to the PN guidance law. On the other hand, when the value of $\beta$ is large, it is close to the first-order sliding-mode guidance law and is robust enough to target maneuvers. However, if the value of $\beta$ is too large, it will cause chattering.

In practical applications, the values of $\eta$ and $\beta$ are to be suitably chosen so as to achieve a compromise of meeting the required convergence rate while alleviating the chattering effect.

\section{Conclusions}

The theory on the finite time stability of nonlinear control systems was used to design finite time convergent guidance laws under which the line-of-sight angular rate converges to zero or a small neighborhood of zero in finite time. The proposed finite time convergent guidance laws were simpler in structure than previous second-order sliding-mode guidance laws. Their complexities were just comparable to those of previous first-order sliding-mode guidance laws. The finite time convergent guidance laws designed in the plane could be directly applied to a three-dimensional guidance process, achieving the finite time convergence of both the elevation angular rate and the azimuth angular rate. The guidance laws with finite time convergence covered the first-order sliding-mode guidance law and they supplied more convenience in selecting the parameters of guidance law. In the case that target acceleration can be estimated, we designed a finite time convergent guidance law with target acceleration compensation. Suitable parameters for this guidance law could ensure finite time convergence while alleviating the chattering effect, even though there exists a signum function in the guidance law. In the case that target acceleration cannot be estimated, we designed a finite time convergent guidance law without target acceleration compensation. This guidance law should be designed to be close to the first-order sliding-mode guidance law such that robustness and rapid convergence rate could be ensured. To remove the chattering, the signum function in the guidance law could be replaced by the saturation function, and then the line-of-sight angular rate converges to a prescribed boundary layer of zero in finite time.

\section{Acknowledgments}

This work is supported in part by the National Natural Scientific Foundation of China (grant number 60674102, 60535010) and the Program for New Century Excellent Talents in University (NCET08-0153). The authors would like to thank Jing-Yang Zhou and especially two anonymous reviewers for their many useful suggestions that substantially improved the quality of this paper.

\section{References}

[1] Guelman, M., "A Qualitative Study of Proportional Navigation," IEEE Transactions on Aerospace and Electronic Systems, Vol. AES-7, No. 4, 1971, pp. 637-643. doi:10.1109/TAES.1971.310406

[2] Guelman, M., "The Closed-Form Solution of True Proportional Navigation," IEEE Transactions on Aerospace and Electronic Systems, Vol. AES-12, No. 4, 1976, pp. 472-482. doi:10.1109/TAES.1976.308328

[3] Yuan, P. J., and Chern, J. S., "Solution of True Proportional Navigation for Maneuvering and Nonmaneuvering Target," Journal of Guidance, Control, and Dynamics, Vol. 15, No. 1, 1992, pp. 268-271. doi: $10.2514 / 3.20828$

[4] Yang, C. D., and Yeh, F. B., "Generalized Guidance Law for Homing Missile," IEEE Transactions on Aerospace and Electronic Systems, Vol. 25, No. 2, 1989, pp. 197-212. doi:10.1109/7.18681

[5] Yuan, P. J., and Hsu, S. C., "Solution of Generalized True Proportional Navigation for Maneuvering and Nonmaneuvering Target," IEEE Transactions on Aerospace and Electronic Systems, Vol. 31, No. 1, 1995, pp. 469-474. doi:10.1109/7.366329

[6] Yuan, P. J., and Chern, J. S., "Ideal Proportional Navigation," Journal of Guidance, Control, and Dynamics, Vol. 15, No. 5, 1992, pp. 11611165.

doi:10.2514/3.20964

[7] Guelman, M., and Shinar, J., "Optimal Guidance Law in the Plane," Journal of Guidance, Control, and Dynamics, Vol. 7, No. 4, 1984, pp. 471-476. 
doi: $10.2514 / 3.19880$

[8] Ha, I. J., Hur, J. S., Ko, M. S., and Song, T. L., "Performance Analysis of PNG Laws for Randomly Maneuvering Targets," IEEE Transactions on Aerospace and Electronic Systems, Vol. 26, No. 5, 1990, pp. 713-721. doi:10.1109/7.102706

[9] Zarchan, P., Tactical and Strategic Missile Guidance, AIAA, Reston, VA, 1997, pp. 161-180

[10] Talole, S. E., and Ravi, N. B., "Proportional Navigation Through Predictive Control," Journal of Guidance, Control, and Dynamics, Vol. 21, No. 6, 1998, pp. 1004-1006. doi: $10.2514 / 2.4339$

[11] Yang, C. D., and Chen, H. Y., "Nonlinear $H_{\infty}$ Robust Guidance Law for Homing Missiles," Journal of Guidance, Control, and Dynamics, Vol. 21, No. 6, 1998, pp. 882-890. doi: $10.2514 / 2.4321$

[12] Zhou, D., Mu, C. D., and Shen, T. L., "Robust Guidance Law with L2 Gain Performance," Transactions of the Japan Society for Aeronautical and Space Sciences, Vol. 44, No. 144, 2001, pp. 82-88. doi: $10.2322 /$ tjsass. 44.82

[13] Lechevin, N., and Rabbath, C. A., "Lyapunov-Based Nonlinear Missile Guidance," Journal of Guidance, Control, and Dynamics, Vol. 27, No. 6, 2004, pp. 1096-1102. doi:10.2514/1.8629

[14] Brierly, S. D., and Longchamp, R., "Application of Sliding Mode Control to Air-Air Interception Problem," IEEE Transactions on Aerospace and Electronic Systems, Vol. 26, No. 2, 1990, pp. 306-325. doi: $10.1109 / 7.53460$

[15] Babu, K. R., Sarma, I. G., and Swamy, K. N., "Switched Bias Proportional Navigation for Homing Guidance Against Highly Maneuvering Target," Journal of Guidance, Control, and Dynamics, Vol. 17, No. 6, 1994, pp. 1357-1363. doi: $10.2514 / 3.21356$

[16] Zhou, D., Mu, C. D., and Xu, W. L., "Adaptive Sliding-Mode Guidance of a Homing Missile," Journal of Guidance, Control, and Dynamics, Vol. 22, No. 4, 1999, pp. 589-594. doi: $10.2514 / 2.4421$

[17] Moon, J., Kim, K., and Kim, Y., "Design of Missile Guidance Law via Variable Structure Control," Journal of Guidance, Control, and Dynamics, Vol. 24, No. 4, 2001, pp. 659-664. doi: $10.2514 / 2.4792$

[18] Haimo, V. T., "Finite Time Controllers," SIAM Journal on Control and Optimization, Vol. 24, No. 4, 1986, pp. 760-770. doi:10.1137/0324047

[19] Shtessel, Y. B., and Buffington, J. M., "Continuous Sliding Mode Control," Proceedings of American Control Conference, American Automatic Control Council, Evanston, IL, 1998, pp. 562-563.

[20] Shtessel, Y. B., and Buffington, J. M., "Finite-Reaching-Time Continuous Sliding Mode Controller for MlMO Nonlinear Systems," Proceedings of the 37th IEEE Conference on Decision and Control, Inst. of Electrical and Electronics Engineers, New York, 1998, pp. 1934-1935.

[21] Bhat, S. P., and Bernstein D. S, "Finite Time Stability and Continuous Autonomous Systems," SIAM Journal on Control and Optimization, Vol. 38, No. 3, 2000, pp. 751-766. doi:10.1137/S0363012997321358

[22] Gurfil, P., Jodorkovsky, M., and Guelman, M., "Finite Time Stability Approach to Proportional Navigation Systems Analysis," Journal of Guidance, Control, and Dynamics, Vol. 21, No. 6, 1998, pp. 853-861. doi: $10.2514 / 2.4348$
[23] Levant, I., "Universal Single-Input-Single-Output (SISO) SlidingMode Controllers with Finite-Time Convergence," IEEE Transactions on Automatic Control, Vol. 46, No. 9, 2001, pp. 1447-1451. doi: 10.1109/9.948475

[24] Hong, Y. G., Huang, J., and Xu, Y. S., "On an Output Feedback FiniteTime Stabilization Problem," IEEE Transactions on Automatic Control, Vol. 46, No. 2, 2001, pp. 305-309. doi:10.1109/9.905699

[25] Hong, Y. G., "Finite Time Stabilization and Stabilizability of a Class of Controllable Systems," Systems \& Control Letters, Vol. 46, No. 4, 2002, pp. 231-236. doi:10.1016/S0167-6911(02)00119-6

[26] Orlov, Y., "Finite Time Stability and Robust Control Synthesis of Uncertain Switched Systems," SIAM Journal on Control and Optimization, Vol. 43, No. 4, 2004, pp. 1253-1271. doi:10.1137/S0363012903425593

[27] Hong, Y. G., Wang, J. K., and Chen, D. Z., "Adaptive Finite-Time Control of Nonlinear Systems with Parametric Uncertainty," IEEE Transactions on Automatic Control, Vol. 51, No. 5, 2006, pp. 858-862. doi:10.1109/TAC.2006.875006

[28] Shkolnikov, I., Shtessel, Y., and Lianos, D., "Integrated GuidanceControl System of Homing Interceptor: Sliding Modes Approach," AIAA Paper 2001-4218, 2001.

[29] Shtessel, Y. B., and Shkolnikov, I. A., "Integrated Guidance and Control of Advanced Interceptors Using Second Order Sliding Modes," Proceedings of the 42th IEEE Conference on Decision and Control, Inst. of Electrical and Electronics Engineers, New York, 2003, pp. 4587-4592.

[30] Marks, G. M., Shtessel, Y. B., and Gratt, H., "Effects of High Order Sliding Mode Guidance and Observers on Hit-to-Kill Interceptions," AIAA Paper 2005-5967, 2005.

[31] Shtessel, Y., Tournes, C., and Shkolnikov, I., "Guidance and Autopilot for Missiles Steered by Aerodynamic Lift and Divert Thrusters Using Second Order Sliding Modes," AIAA Paper 2006-6784, 2006.

[32] Song, S. H., and Ha, I. J., "A Lyapunov-Like Approach to Performance Analysis of 3-Dimensional Pure PNG Laws," IEEE Transactions on Aerospace and Electronic Systems, Vol. 30, No. 1, 1994, pp. 238-247. doi:10.1109/7.250424

[33] Yang, C. D., and Yang, C. C., "Analytical Solution of ThreeDimensional Realistic True Proportional Navigation," Journal of Guidance, Control, and Dynamics, Vol. 19, No. 3, 1996, pp. 569-577. doi: $10.2514 / 3.21659$

[34] Yang, C. D., and Yang, C. C., "Analytical Solution of Generalized Three-Dimensional Proportional Navigation," Journal of Guidance, Control, and Dynamics, Vol. 19, No. 3, 1996, pp. 721-724. doi: $10.2514 / 3.21685$

[35] Yang, C. D., and Yang, C. C., "Analytical Solution of 3D True Proportional Navigation," IEEE Transactions on Aerospace and Electronic Systems, Vol. 32, No. 4, 1996, pp. 1509-1522. doi: $10.1109 / 7.543873$

[36] Oh, J. H., and Ha, I. J., "Capturability of the 3-Dimensional Pure PNG Law," IEEE Transactions on Aerospace and Electronic Systems, Vol. 35, No. 2, 1999, pp. 491-503. doi: $10.1109 / 7.766931$

[37] Tyan, F., "Unified Approach to Missile Guidance Laws: A 3D Extension," IEEE Transactions on Aerospace and Electronic Systems, Vol. 41, No. 4, 2005, pp. 1178-1199. doi: 10.1109/TAES.2005.1561882 